Received: 2015.07.07

Accepted: 2015.08.13

Published: 2015.10.21

\title{
Common Polymorphisms in the NFKBIA Gene and Cancer Susceptibility: A Meta-Analysis
}

Authors' Contribution: Study Design A Data Collection B Statistical Analysis C Data Interpretation D Manuscript Preparation E Literature Search F Funds Collection G
Corresponding Authors: Source of support:
AB 1 Meng Zhang*

EF 2 Junjie Huang*

B 1 Xiuxiu Tan*

D 1 Jian Bai

BC 1 Hao Wang

DE 1 Yukun Ge

CD 1 Hu Xiong

BE 3 Jizhou Shi

CD 1 Wei Lu

BEG 1 Zhaojie Lv

BCDE 4,5 Chaozhao Liang
1 Department of Urology, Shenzhen Second People's Hospital, Clinical Medicine College of Anhui Medical University, Shenzhen, Anhui, P.R. China

2 School of Medicine, Shenzhen University, Shenzhen, Anhui, P.R. China

3 Department of Medicine, Shengli Oilfield Central Hospital, Dongying, Shandong, P.R. China

4 Department of Urology, The First Affiliated Hospital of Anhui Medical University, Hefei, Anhui, P.R. China

5 Institute of Urology, Anhui Medical University, Hefei, Anhui, P.R. China.

Background: $\quad$ NFKBIA encodes the inhibitors of nuclear factor- $\mathrm{KB}(\mathrm{NF}-\mathrm{KB})$, which regulate the translation of the genes involved in the inflammatory and immune reactions. Polymorphisms (rs2233406, rs3138053, and rs696) of NFKBIA have been implicated in susceptibility to many cancer types.

Material/Methods: To evaluate the association between polymorphisms of NFKBIA and cancer susceptibility, a meta-analysis including a total of 7182 cancer cases and 10057 controls from 28 case-control studies was performed. Data were extracted and pooled odds ratios (ORs) with $95 \%$ confidence intervals (Cls) were calculated.

Results: Combined data demonstrated that rs 3138053 polymorphism of NFKBIA was associated with cancer susceptibility in an allelic model (C vs. T: OR=10.754, 95\%Cl=4.175-27.697, $\left.P_{\text {heterogeneity }}=0.000\right)$, while the polymorphism of rs696 appeared to play a protective role in tumorigenesis $\left(C C+C T\right.$ vs. TT: OR=0.879, 95\% $\left.\mathrm{Cl}=0.787-0.982, P_{\text {heteroeneity }}=0.107\right)$. When stratification analysis was performed by cancer type, an increased association of rs 3138053 was recognized in hepatocarcinoma ( $C$ vs. T: OR=42.180, 95\% Cl=27.970-63.612, $P_{\text {heterogeneity }}=0.007$ ), while a decreased association of rs696 was identified in Hodgkin lymphoma (C vs. T: OR=0.792, 95\%Cl=0.656-0.956, $P_{\text {heterogeneity }}=0.116$; CC vs. TT: $\mathrm{OR}=0.658,95 \% \mathrm{Cl}=0.448-0.965, P_{\text {heterogeneity }}=0.076$; CC vs. $\mathrm{CT}+\mathrm{TT}: \mathrm{OR}=0.734,95 \% \mathrm{Cl}=0.562-0.958$, $P_{\text {heterogeneity }}=0.347$ ). By ethnicity, rs696 appears to be a protective candidate among Caucasians (CT vs. TT: $\mathrm{OR}=0.809,95 \% \mathrm{Cl}=0.676-0.969, P_{\text {heterogeneity }}=0.459$ ).

Conclusions: Our data demonstrated that the rs 3138053 polymorphism of NFKBIA gene is a candidate for susceptibility to overall cancers, while rs696 plays a protective role.

MeSH Keywords: $\quad$ Genetic Linkage $\bullet$ Medical Oncology $\bullet$ Meta-Analysis

Full-text PDF: http://www.medscimonit.com/abstract/index/idArt/895257

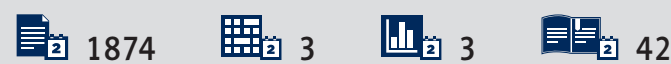




\section{Background}

Nuclear factor- $\mathrm{KB}$ (NF- $\mathrm{KB}$ ) belongs to a family of transcription factors that play a crucial role in inflammatory and immune reactions [2]. The malfunctioning of NF- $\mathrm{KB}$ contributes to the inhibition of apoptosis, cell replication, and angiogenesis, all of which are occur in cancer cells [3]. Several pieces of evidence have demonstrated the connection between the defective function of IKB and cancer progression. Overexpression of NF-KB has been identified in several categories of cancer, including Hodgkin's lymphoma, multiple myeloma, colorectal cancer, and melanoma [1]. Additionally, it was discovered that the expression of IKB was decreased among prostate cancer patients [4]. These results indicate the significant role of $1 \mathrm{KBs}$ in regulating the oncogenic potential of NF-KB and in cancer development. From these points of view, malfunctioning in the expression of IKB may remain a risk factor for cancer.

I $\mathrm{KB} \alpha$ is encoded by NFKBIA genes located on chromosome 14q13. NFKBIA rs2233406, rs3138053, and rs696 polymorphisms are situated in the binding regions for CCAAT/enhancer binding protein and GATA binding protein 2, respectively. They may regulate IKB $\alpha$ expression, and influence NF- $\mathrm{KB}$ activation; these polymorphisms (rs2233406, rs3138053, and rs696) are directly related to apoptosis, inappropriate immune cell development, and delayed cell growth [5]. The effect of polymorphisms within the NFKBIA gene on cancer susceptibility has been investigated in a number of cancers [6-12]. It was reported that polymorphic variants in the 39-untranslated region of NFKBIA was associated with a susceptibility to multiple myeloma, Hodgkin's lymphoma, prostate cancer, breast cancer, colorectal cancer, gastric cancer, and melanoma [13-19].

However, the susceptibility modulation impacts of the polymorphisms were inconsistent in various studies because the sample sizes enrolled were limited and the ethnic backgrounds of subjects in various studies were different. Evidence of the relationship between genetic polymorphisms and cancer susceptibility can be provided by a quantitative synthesis to accumulate data from different studies. In this paper we present the results of a comprehensive meta-analysis performed on publicly available databases.

\section{Material and Methods}

\section{Literature sources and search strategy}

We conducted a systematic literature search in Google Scholar, PubMed, and Web of Science databases (up to 20 June 2015) to accumulate all available studies on the association between polymorphisms of NFKBIA (rs2233406, rs3138053, and rs696) and cancer susceptibility by using the following the search strategy: ("NFKBIA" OR "Nuclear factor kappa B inhibitor") AND ("polymorphism" OR "mutation" OR "variation") AND ("susceptibility" OR "risk" OR "effects") AND ("cancer" OR "tumour" OR "carcinoma"). Studies were also searched manually on the reference lists of reviews and retrieved studies for additional eligible studies.

\section{Inclusion and exclusion criteria}

The articles enrolled in the present meta-analysis were consistent with these criteria: (a) the relationship between the polymorphisms in NFKBIA and cancer susceptibility was identified in the studies; (b) the study method was case-control; and (c) we could extract the ORs with $95 \% \mathrm{Cls}$ of all the cases and controls. Studies were excluded when they were: (a) studies without sufficient raw data to evaluate odds ratios with 95\% confidence intervals; (b) case-only studies; (c) duplicated publications; and (d) studies based on animals or families.

\section{Data extraction}

The data were extracted independently by 3 investigators (M. Zhang, J. J. Huang, and X. X. Tan). Data with discrepancies were discussed by all authors. The following data were collected: name of first author, publication year, country of origin, ethnicity, cancer type, total numbers of cases and controls, source of controls, and genotype or allele distribution in cases and controls. Ethnic backgrounds were categorized as Asian and Caucasian.

\section{Statistical analysis}

We assessed the relationship between the NFKBIA polymorphisms and cancer susceptibility by employing the ORs and 95\% Cls in the studies and calculated the pooled ORs on the allele contrast ( $\mathrm{t} v \mathrm{~s} . \mathrm{T})$, dominant ( $\mathrm{Tt}+\mathrm{tt}$ vs. TT), and recessive ( $\mathrm{tt}$ vs. $\mathrm{Tt}+\mathrm{TT}$ ) models. Comparisons were also performed in heterozygote (Tt vs. TT) and homozygote (tt vs. TT) (TT, homozygotes for the common allele; Tt, heterozygotes; tt, homozygotes for the rare allele). The $P$ values of HWE were calculated by $\chi^{2}$ test for the genotype distribution in controls. The meta-analyses were conducted by using STATA 12.0 software (Stata Corporation, College Station, Texas). A chi-square based Q-statistic test was performed to evaluate the heterogeneity of studies in the case-control studies [20]. If the $Q$ test $(P>0.1)$ indicated homogeneity within studies, the fixedeffects model was used [21]; otherwise, the random-effects model was used [22]. We also evaluated heterogeneity across studies by calculation of the inconsistency index $\left(1^{2}<25 \%\right.$ : no heterogeneity; $I^{2}=25-50 \%$ : moderate heterogeneity; $I^{2}>50 \%$ : significant heterogeneity). Stratification analyses were performed by source of control, cancer type, and ethnicity. We removed a single study each time to evaluate the stability of 


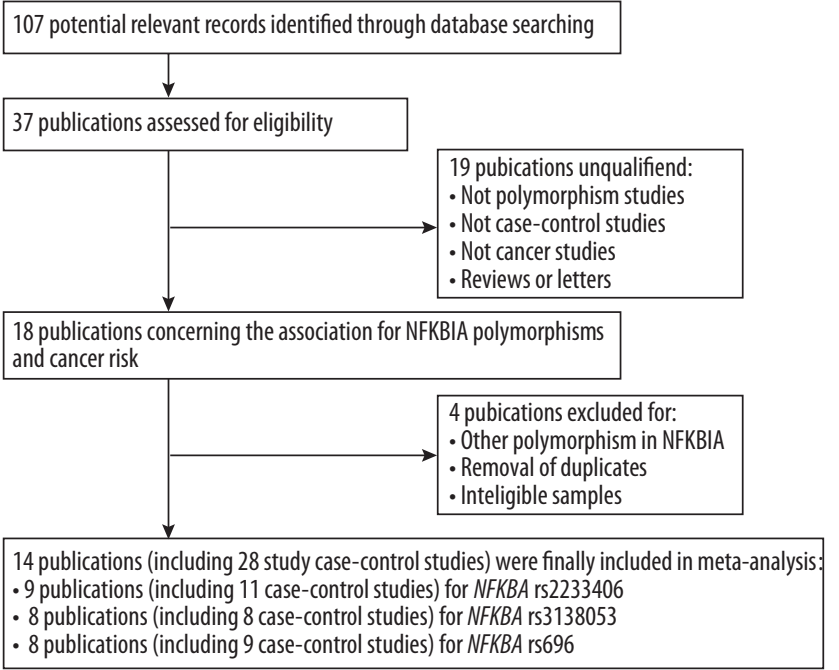

Figure 1. Flow chart presenting the study selection procedure.

the results. Begg's funnel plot and Egger's test were used to assess publication bias.

\section{Results}

\section{The identification and characteristics of eligible studies}

As demonstrated in Figure 1, after a systematic literature search in the databases on the relevance between NFKBIA polymorphisms and cancer susceptibility, a total of 107 potential records were initially identified. After checking the abstracts, 70 irrelevant studies were excluded, some studies were with insufficient data and others were duplicated studies. When the full texts were examined, we excluded 19 articles with no polymorphism studies, non-case-control studies, studies not on cancer, and reviews. Another 4 publications were excluded because they were on other polymorphisms in NFKBIA, were duplicates, or lacked eligible samples. Finally, 14 articles containing 28 independent case-control studies with a total of 7182 cases and 10057 controls were enrolled in this meta-analysis [23-36]. Table 1 presents the characteristics of all eligible studies; 9 were population-based and the others were hospitalbased. All studies were case-controlled, including 9 liver cancer studies, 5 colorectal cancer studies, 3 breast cancer studies, 3 prostate cancer studies, 2 oral cancer studies, 2 oesophageal cancer studies, 1 ovarian cancer study, and 1 multiple myeloma study. The ethnicities in these case-control studies were categorized as Asian (23 studies) and Caucasian (5 studies).

\section{Pooled analysis}

The primary results of the present meta-analysis and the heterogeneity test are summarized in Table 2 . In addition, we also rated the methodological quality of the included studies according to the Newcastle-Ottawa Scale (Table 3). By pooling ORs and $95 \% \mathrm{Cls}$, we discovered that rs2233406 polymorphism of NFKBIA was not associated with susceptibility to cancers (Table 2A). However, we identified a significant increased susceptibility in the rs3138053 polymorphism of NFKBIA (C vs. T: $\mathrm{OR}=10.754,95 \% \mathrm{Cl}=4.175-27.697, P_{\text {heterogeneity }}=0.000$; Figure $2 \mathrm{~A}$, Table 2B). Another impressive finding was that the polymorphism of rs696 appeared to play a protective role in tumorigenesis, as suggested by the pooled ORs (CC+CT vs. TT: OR=0.879, $95 \% \mathrm{Cl}=0.787-0.982, P_{\text {heterogeneity }}=0.107$; Figure 2B, Table 2C).

\section{Subgroup analysis}

In the subgroup meta-analysis by cancer type, the rs3138053 polymorphism of NFKBIA was revealed to be an important factor in HCC cancer susceptibility, and the pooled results were statistically significant (C vs. T: $\mathrm{OR}=42.180,95 \% \mathrm{Cl}=27.970-63.612$, $P_{\text {heterogeneity }}=0.007$; Table 2B). Some significantly decreased susceptibility of the rs696 polymorphism of NFKBIA was observed in Hodgkin lymphoma (C vs. T: OR=0.792, 95\%Cl=0.656-0.956, $P_{\text {heterogeneity }}=0.116$; CC vs. TT: OR $=0.658,95 \% \mathrm{Cl}=0.448-0.965$, $P_{\text {heterogeneity }}=0.076 ;$ CC vs. CT+TT: OR=0.734, $95 \% \mathrm{Cl}=0.562-0.958$, $P_{\text {heterogeneity }}=0.347$; Table $2 \mathrm{C}$ ). The source analysis indicated positive association of the rs3138053 polymorphism in the hospital-based group (C vs. T: OR=10.381, 95\% Cl=3.513-30.677, $P_{\text {heterogeneity }}=0.000 ; \mathrm{CC}+\mathrm{TC}$ vs. TT: $\mathrm{OR}=1.405,95 \% \mathrm{Cl}=1.146-1.721$, $P_{\text {heterogeneity }}=0.114 ; \mathrm{CC}$ vs. TC+TT: $\mathrm{OR}=2.460,95 \% \mathrm{Cl}=1.686-3.590$, $P_{\text {heterogeneity }}=0.867$; Table $2 \mathrm{~B}$ ) and the population-based group (C vs. T: OR=11.377, $95 \% \mathrm{Cl}=1.472-87.963, P_{\text {heterogeneity }}=0.000$; Table 2B). Caucasians seems to benefit more from the polymorphism of rs696 (CT vs. TT: OR=0.809, 95\% Cl=0.676$0.969, P_{\text {heterogeneity }}=0.459$; Table $2 \mathrm{C}$ ) than Asians (CT vs. TT: $\mathrm{OR}=0.921,95 \% \mathrm{Cl}=0.691-1.227, P_{\text {heterogeneity }}=0.015$; Table $2 \mathrm{C}$ ). 
Table 1. Characteristics of the enrolled studies.

\begin{tabular}{|c|c|c|c|c|c|c|c|c|c|c|c|c|c|c|}
\hline \multirow[b]{2}{*}{ SNP } & \multirow{2}{*}{$\begin{array}{l}\text { First } \\
\text { author }\end{array}$} & \multirow[b]{2}{*}{ Year } & \multirow[b]{2}{*}{ Ethnicity } & \multirow{2}{*}{$\begin{array}{l}\text { Genotyping } \\
\text { method }\end{array}$} & \multirow{2}{*}{$\begin{array}{c}\text { Source } \\
\text { of } \\
\text { control }\end{array}$} & \multirow{2}{*}{$\begin{array}{l}\text { Cancer } \\
\text { type }\end{array}$} & \multicolumn{5}{|c|}{ Control } & \multicolumn{3}{|c|}{ Case } \\
\hline & & & & & & & AA & AB & BB & $\begin{array}{c}P \\
\text { (HWE) }\end{array}$ & $\mathbf{Y}$ or $\mathbf{N}$ & $\mathbf{A A}$ & $\mathbf{A B}$ & BB \\
\hline \multirow[t]{11}{*}{ rs2233406 } & Lu et al. & 2015 & Asian & PCR-RFLP & $\mathrm{HB}$ & OC & 478 & 190 & 19 & 0.982 & Y & 486 & 181 & 20 \\
\hline & Zhang et al. & 2014 & Asian & PCR & $\mathrm{HB}$ & $\mathrm{HCC}$ & 1292 & 321 & 28 & 0.123 & Y & 204 & 41 & 6 \\
\hline & Cheng et al. & 2013 & Asian & PCR & $\mathrm{HB}$ & $\mathrm{HCC}$ & 438 & 78 & 4 & 0.797 & Y & 106 & 27 & 2 \\
\hline & Lin et al. & 2012 & Asian & TaqMan & $\mathrm{HB}$ & ORC & 438 & 78 & 4 & 0.797 & Y & 351 & 101 & 10 \\
\hline & Tan et al. & 2013 & Asian & PCR & $\mathrm{HB}$ & CRC & 163 & 69 & 5 & 0.459 & Y & 169 & 60 & 8 \\
\hline & Wang et al. & 2014 & Asian & PCR-RFLP & $\mathrm{HB}$ & $B C$ & 297 & 162 & 42 & 0.004 & N & 212 & 102 & 32 \\
\hline & Wang et al. & 2014 & Asian & PCR-RFLP & $\mathrm{HB}$ & $B C$ & 297 & 162 & 42 & 0.004 & N & 46 & 25 & 0 \\
\hline & Wang et al. & 2014 & Asian & PCR-RFLP & $\mathrm{HB}$ & $B C$ & 297 & 162 & 42 & 0.004 & $\mathrm{~N}$ & 30 & 20 & 7 \\
\hline & He et al. & 2009 & Asian & PCR & $\mathrm{HB}$ & $\mathrm{HCC}$ & 685 & 181 & 20 & 0.056 & Y & 149 & 52 & 1 \\
\hline & Han et al. & 2015 & Asian & PCR-RFLP & $\mathrm{HB}$ & PC & 586 & 321 & 29 & 0.058 & $Y$ & 508 & 356 & 72 \\
\hline & Umar et al. & 2013 & Asian & PCR & PB & $\mathrm{ESCC}$ & 149 & 141 & 21 & 0.106 & Y & 145 & 122 & 23 \\
\hline \multirow[t]{8}{*}{ rs3138053 } & Lin et al. & 2012 & Asian & TaqMan & $\mathrm{HB}$ & ORC & 438 & 78 & 4 & 0.797 & Y & 351 & 101 & 10 \\
\hline & Tan et al. & 2013 & Asian & PCR & $\mathrm{HB}$ & CRC & 163 & 69 & 5 & 0.459 & $Y$ & 169 & 60 & 8 \\
\hline & Gao et al. & 2014 & Asian & PCR & PB & $\mathrm{HCC}$ & 336 & 48 & 40 & 0.000 & N & 173 & 21 & 19 \\
\hline & Spink et al. & 2006 & Caucasian & PCR & PB & MM & 94 & 91 & 11 & 0.066 & Y & 64 & 77 & 16 \\
\hline & He et al. & 2009 & Asian & PCR & $\mathrm{HB}$ & $\mathrm{HCC}$ & 780 & 106 & 0 & 0.058 & Y & 164 & 38 & 0 \\
\hline & Han et al. & 2015 & Asian & PCR-RFLP & $\mathrm{HB}$ & PC & 586 & 321 & 29 & 0.058 & Y & 508 & 356 & 72 \\
\hline & Zhang et al. & 2014 & Asian & PCR & PB & $\mathrm{HCC}$ & 1289 & 308 & 27 & 0.087 & Y & 214 & 38 & 1 \\
\hline & Cheng et al. & 2013 & Asian & PCR & $\mathrm{HB}$ & $\mathrm{HCC}$ & 438 & 78 & 4 & 0.797 & Y & 106 & 27 & 2 \\
\hline \multirow[t]{9}{*}{ rs696 } & Umar et al. & 2013 & Asian & PCR & PB & ESCC & 59 & 165 & 87 & 0.219 & Y & 71 & 140 & 79 \\
\hline & Gao et al. & 2006 & Asian & PCR-RFLP & $\mathrm{HB}$ & CRC & 81 & 259 & 237 & 0.450 & Y & 26 & 109 & 64 \\
\hline & Gao et al. & 2006 & Caucasian & PCR-RFLP & $\mathrm{HB}$ & CRC & 74 & 221 & 143 & 0.466 & Y & 29 & 72 & 54 \\
\hline & Zhang et al. & 2014 & Asian & PCR & $\mathrm{HB}$ & $\mathrm{HCC}$ & 248 & 794 & 561 & 0.231 & Y & 55 & 109 & 84 \\
\hline & Gao et al. & 2014 & Asian & HapMap & PB & $\mathrm{HCC}$ & 149 & 196 & 76 & 0.412 & Y & 65 & 115 & 33 \\
\hline & Osborne et al. & 2005 & Caucasian & PCR & PB & $\mathrm{HL}$ & 8 & 22 & 20 & 0.64 & Y & 4 & 26 & 21 \\
\hline & Chang et al. & 2009 & Caucasian & Taqman & PB & $\mathrm{HL}$ & 53 & 158 & 153 & 0.245 & Y & 92 & 215 & 156 \\
\hline & Han et al. & 2015 & Asian & PCR-RFLP & $\mathrm{HB}$ & PC & 165 & 458 & 313 & 0.909 & Y & 173 & 442 & 321 \\
\hline & Song et al. & 2011 & Caucasian & PCR-RFLP & PB & CRC & 212 & 531 & 262 & 0.06 & $Y$ & 233 & 460 & 308 \\
\hline
\end{tabular}

PCR - polymerase chain reaction; PCR-RFLP - polymerase chain reaction-restriction fragment length polymorphism; Y $-P_{(H W E)}>0.05$; $\mathrm{N}-P_{(\mathrm{HWE})} \leq 0.05$; HCC - hepatocellular carcinoma; OC - ovarian cancer; BC - breast cancer; CRC - colorectal cancer; $\mathrm{HL}$ - Hodgkin lymphoma; PC - prostate cancer; ESCC - esophageal squamous cell carcinoma; MM - multiple myeloma; ORC - oral cancer; HB - hospital-based; PB - population-based;

In addition, polymorphisms that conformed to HWE in the control group showed positive association in rs2233406 (CC vs. $\mathrm{CT}+\mathrm{TT}: \mathrm{OR}=1.535,95 \% \mathrm{Cl}=1.027-2.296, P_{\text {heterogeneity }}=0.099$; Figure 2C and Table 2A) and rs3138053 (CC vs. TT: OR=2.133, $95 \% \mathrm{Cl}=1.317-3.455, P_{\text {heterogeneity }}=0.217 ; \mathrm{CC}$ vs. TC $+\mathrm{TT}: \mathrm{OR}=2.063$, $95 \% \mathrm{Cl}=1.350-3.154, P_{\text {heterogeneity }}=0.296$; Table $2 \mathrm{~B}$ ).

\section{Sensitivity analysis and publication bias risk}

The sensitivity analyses were conducted by excluding each single case-control study in turn, and no separate study shows an influence on the pooled OR. Begg's funnel plot and Egger's test were performed to assess the risk of publication bias and no visual publication bias was shown (rs3138053: $C$ vs. T: $P=0.181$ for egger's test; rs696: CC+CT vs. TT: $P=0.552$ for 
Table 2A. Results of meta-analysis for rs2233406 polymorphism in NFKBA and cancer susceptibility.

\begin{tabular}{|c|c|c|c|c|c|c|c|c|c|c|}
\hline \multirow{2}{*}{$\begin{array}{c}\text { Variables } \\
\text { (rs2233406) }\end{array}$} & \multirow{2}{*}{$\begin{array}{l}\text { Case/ } \\
\text { control }\end{array}$} & \multicolumn{3}{|c|}{ C vs. T } & \multicolumn{3}{|c|}{ CC vs. TT } & \multicolumn{3}{|c|}{ CT vs. TT } \\
\hline & & OR $(95 \% \mathrm{CI})$ & $p^{a}$ & $I^{2}(\%)$ & OR $(95 \% \mathrm{Cl})$ & $p^{a}$ & $I^{2}(\%)$ & OR $(95 \% \mathrm{Cl})$ & $p^{a}$ & $I^{2}(\%)$ \\
\hline Total & $3674 / 7241$ & $\begin{array}{c}1.098 \\
(0.944-1.277)\end{array}$ & 0.000 & 47.1 & $\begin{array}{c}1.387 \\
(0.942-2.042)\end{array}$ & 0.017 & 28.9 & $\begin{array}{c}1.077 \\
(0.931-1.246)\end{array}$ & 0.030 & 24.8 \\
\hline \multicolumn{11}{|c|}{ Source of control } \\
\hline $\mathrm{HB}$ & $3384 / 6930$ & $\begin{array}{c}1.112 \\
(0.942-1.312)\end{array}$ & 0.000 & 49.4 & $\begin{array}{c}1.417 \\
(0.914-2.198)\end{array}$ & 0.014 & 32.1 & $\begin{array}{c}1.099 \\
(0.941-1.284)\end{array}$ & 0.031 & 25.9 \\
\hline \multicolumn{11}{|l|}{ Cancer type } \\
\hline $\mathrm{HCC}$ & $588 / 3047$ & $\begin{array}{c}1.082 \\
(0.851-1.375)\end{array}$ & 0.236 & 9.5 & $\begin{array}{c}1.038 \\
(0.348-3.103)\end{array}$ & 0.187 & 16.3 & $\begin{array}{c}1.131 \\
(0.789-1.622)\end{array}$ & 0.082 & 35.8 \\
\hline$B C$ & $474 / 1503$ & $\begin{array}{c}0.841 \\
(0.581-1.215)\end{array}$ & 0.132 & 31.2 & $\begin{array}{c}0.390 \\
(0.026-5.968)\end{array}$ & 0.048 & 55.2 & $\begin{array}{c}1.222 \\
(0.673-2.221)\end{array}$ & 0.693 & 0.0 \\
\hline \multicolumn{11}{|l|}{ HWE } \\
\hline Y & $3200 / 5738$ & $\begin{array}{c}1.148 \\
(0.967-1.362)\end{array}$ & 0.002 & 48.9 & $\begin{array}{c}1.535 \\
(0.982-2.400)\end{array}$ & 0.046 & 26.1 & $\begin{array}{c}1.103 \\
(0.924-1.318)\end{array}$ & 0.014 & 36.2 \\
\hline \multirow[t]{3}{*}{$\mathrm{N}$} & $474 / 1503$ & $\begin{array}{c}0.952 \\
(0.691-1.310)\end{array}$ & 0.096 & 32.9 & $\begin{array}{c}0.994 \\
(0.398-2.484)\end{array}$ & 0.084 & 35.5 & $\begin{array}{c}0.954 \\
(0.750-1.214)\end{array}$ & 0.624 & 0.0 \\
\hline & \multirow{2}{*}{$\begin{array}{l}\text { Casel } \\
\text { control }\end{array}$} & \multicolumn{3}{|c|}{ CC+CT vs. TT } & \multicolumn{3}{|c|}{ CC vs. CT+TT } & & & \\
\hline & & OR $(95 \% \mathrm{Cl})$ & $p^{a}$ & $I^{2}(\%)$ & OR $(95 \% \mathrm{CI})$ & $p^{a}$ & $I^{2}(\%)$ & & & \\
\hline Total & $3674 / 7241$ & $\begin{array}{c}1.097 \\
(0.937-1.283)\end{array}$ & 0.005 & 36.2 & $\begin{array}{c}1.390 \\
(0.977-1.978)\end{array}$ & 0.043 & 21.8 & & & \\
\hline \multicolumn{11}{|l|}{ Source of control } \\
\hline HB & $3384 / 6930$ & $\begin{array}{c}1.117 \\
(0.944-1.323)\end{array}$ & 0.005 & 37.9 & $\begin{array}{c}1.409 \\
(0.940-2.111)\end{array}$ & 0.032 & 25.7 & & & \\
\hline \multicolumn{11}{|l|}{ Cancer type } \\
\hline $\mathrm{HCC}$ & $588 / 3047$ & $\begin{array}{c}1.115 \\
(0.821-1.515)\end{array}$ & 0.146 & 23.0 & $\begin{array}{c}1.014 \\
(0.324-3.175)\end{array}$ & 0.165 & 19.9 & & & \\
\hline BC & $474 / 1503$ & $\begin{array}{c}0.889 \\
(0.695-1.138)\end{array}$ & 0.616 & 0.0 & $\begin{array}{c}0.396 \\
(0.024-6.446)\end{array}$ & 0.043 & 57.0 & & & \\
\hline \multicolumn{11}{|l|}{ HWE } \\
\hline $\mathrm{Y}$ & $3200 / 5738$ & $\begin{array}{c}1.137 \\
(0.944-1.369)\end{array}$ & 0.004 & 43.7 & $\begin{array}{c}1.535 \\
(1.027-2.296)^{*}\end{array}$ & 0.099 & 17.6 & & & \\
\hline $\mathrm{N}$ & $474 / 1503$ & $\begin{array}{c}0.949 \\
(0.758-1.188)\end{array}$ & 0.398 & 0.0 & $\begin{array}{c}1.009 \\
(0.425-2.396)\end{array}$ & 0.094 & 33.3 & & & \\
\hline
\end{tabular}

Egger's test, Figure 3A; rs2233406: CC vs. CT+TT: $P=0.175$ for Egger's test, Figure $3 \mathrm{~B}$ ).

\section{Discussion}

The activation and translocation of NF- $\mathrm{KB}$ to the nucleus modulate the translation of the genes involved in inflammatory and immune activities, cell adhering, differentiating, growing, angiogenesis, and apoptosis through kinases, which leads to the phosphorylation, ubiquitination, and degradation of IKBs [37]. The p50 subunit, encoded by the NF- $\mathrm{KB}$, has several common polymorphisms in the promoter region. The promoter sequence polymorphisms contribute to an increased expression of NF- $\kappa B$ messenger $(m)$ RNA. NF- $K B$ is important to cancer pathogenesis, preventing apoptosis and enhancing growth and survival by the upregulation of several genes [38]. Individual singlenucleotide polymorphisms (rs2233406, rs3138053, and rs696) in the NFKBIA gene may affect expression and function of the protein. Specifically, allelic differences in the NFKBIA promoter and 30UTR region may change I $\mathrm{KB}$ a expression and affect complex formation with NF- $\mathrm{KB}$. In this way, cell growth and anti-apoptosis are regulated [39].

Li et al. [40] and Zou et al. [41] reported that genetic polymorphisms of the NFKBIA gene were associated with cancer 
Table 2B. Results of meta-analysis for rs3138053 polymorphism in NFKBA and cancer susceptibility.

\begin{tabular}{|c|c|c|c|c|c|c|c|c|c|c|}
\hline \multirow{2}{*}{$\begin{array}{c}\text { Variables } \\
\text { (rs3138053) }\end{array}$} & \multirow{2}{*}{$\begin{array}{l}\text { Case/ } \\
\text { control }\end{array}$} & \multicolumn{3}{|c|}{ C vs. T } & \multicolumn{3}{|c|}{ CC vs. TT } & \multicolumn{3}{|c|}{ TC vs. TT } \\
\hline & & OR $(95 \% \mathrm{Cl})$ & $p^{a}$ & $I^{2}(\%)$ & OR (95\% CI) & $p^{a}$ & $I^{2}(\%)$ & OR $(95 \% \mathrm{CI})$ & $p^{a}$ & $I^{2}(\%)$ \\
\hline Total & $2595 / 5343$ & $\begin{array}{c}10.754 \\
(4.175-27.697)^{\star}\end{array}$ & 0.000 & 97.8 & $\begin{array}{c}1.683 \\
(0.979-2.891)\end{array}$ & 0.023 & 34.8 & $\begin{array}{c}1.178 \\
(0.954-1.455)\end{array}$ & 0.012 & 39.9 \\
\hline \multicolumn{11}{|l|}{ Source of control } \\
\hline $\mathrm{HB}$ & $1972 / 3099$ & $\begin{array}{c}10.381 \\
(3.513-30.677)^{\star}\end{array}$ & 0.000 & 97.4 & $\begin{array}{c}2.652 \\
(1.810-3.886)\end{array}$ & 0.767 & 0.0 & $\begin{array}{c}1.335 \\
(1.075-1.657)\end{array}$ & 0.093 & 24.7 \\
\hline PB & $623 / 2244$ & $\begin{array}{c}11.377 \\
(1.472-87.963)^{\star}\end{array}$ & 0.000 & 98.6 & $\begin{array}{c}1.029 \\
(0.415-2.551)\end{array}$ & 0.068 & 39.3 & $\begin{array}{c}0.913 \\
(0.662-1.259)\end{array}$ & 0.205 & 13.5 \\
\hline \multicolumn{11}{|l|}{ Ethnicity } \\
\hline Asian & $2438 / 5147$ & $\begin{array}{c}13.628 \\
(4.922-37.731)\end{array}$ & 0.000 & 97.8 & $\begin{array}{c}1.577 \\
(0.817-3.043)\end{array}$ & 0.013 & 42.9 & $\begin{array}{c}1.168 \\
(0.920-1.483)\end{array}$ & 0.006 & 44.5 \\
\hline \multicolumn{11}{|l|}{ Cancer type } \\
\hline $\mathrm{HCC}$ & $590 / 3030$ & $\begin{array}{c}42.180 \\
(27.970-63.612)^{*}\end{array}$ & 0.007 & 63.8 & $\begin{array}{c}0.718 \\
(0.070-7.340)\end{array}$ & 0.077 & 46.2 & $\begin{array}{c}1.206 \\
(0.706-2.062)\end{array}$ & 0.007 & 63.7 \\
\hline \multicolumn{11}{|l|}{ HWE } \\
\hline Y & $2382 / 4919$ & $\begin{array}{c}10.585 \\
(3.663-30.581)\end{array}$ & 0.000 & 98.0 & $\begin{array}{c}2.133 \\
(1.317-3.455)^{*}\end{array}$ & 0.217 & 8.5 & $\begin{array}{c}1.216 \\
(0.975-1.518)\end{array}$ & 0.012 & 40.1 \\
\hline \multirow[t]{3}{*}{$\mathrm{N}$} & $213 / 424$ & $\begin{array}{c}12.036 \\
(8.430-17.184)\end{array}$ & - & - & $\begin{array}{c}0.923 \\
(0.519-1.641)\end{array}$ & - & - & $\begin{array}{c}0.850 \\
(0.493-1.465)\end{array}$ & - & - \\
\hline & \multirow{2}{*}{$\begin{array}{l}\text { Case/ } \\
\text { control }\end{array}$} & \multicolumn{3}{|c|}{ CC+TC vs. TT } & \multicolumn{3}{|c|}{ CC vs. TC+TT } & & & \\
\hline & & OR $(95 \% \mathrm{Cl})$ & $p^{a}$ & $I^{2}(\%)$ & OR $(95 \% \mathrm{CI})$ & $\boldsymbol{P}^{\mathbf{a}}$ & $I^{2}(\%)$ & & & \\
\hline Total & $2595 / 5343$ & $\begin{array}{c}1.209 \\
(0.964-1.517)\end{array}$ & 0.002 & 48.9 & $\begin{array}{c}1.632 \\
(1.001-2.660)\end{array}$ & 0.055 & 26.3 & & & \\
\hline \multicolumn{11}{|c|}{ Source of control } \\
\hline $\mathrm{HB}$ & $1972 / 3099$ & $\begin{array}{c}1.405 \\
(1.146-1.721)^{\star}\end{array}$ & 0.114 & 21.4 & $\begin{array}{c}2.460 \\
(1.686-3.590)^{*}\end{array}$ & 0.867 & 39.0 & & & \\
\hline PB & $623 / 2244$ & $\begin{array}{c}0.928 \\
(0.639-1.347)\end{array}$ & 0.074 & 37.9 & $\begin{array}{c}1.632 \\
(1.001-2.660)\end{array}$ & 0.105 & 26.3 & & & \\
\hline \multicolumn{11}{|l|}{ Ethnicity } \\
\hline Asian & $2438 / 5147$ & $\begin{array}{c}1.192 \\
\left(0.923 \_1.539\right)\end{array}$ & 0.001 & 54.9 & $\begin{array}{c}1.553 \\
(0.851-2.834)\end{array}$ & 0.031 & 35.2 & & & \\
\hline \multicolumn{11}{|l|}{ Cancer type } \\
\hline $\mathrm{HCC}$ & $590 / 3030$ & $\begin{array}{c}1.192 \\
(0.668-2.127)\end{array}$ & 0.003 & 69.1 & $\begin{array}{c}0.716 \\
(0.080-6.438)\end{array}$ & 0.094 & 41.3 & & & \\
\hline \multicolumn{11}{|l|}{ HWE } \\
\hline$Y$ & $2382 / 4919$ & $\begin{array}{c}1.260 \\
(0.992-1.601)\end{array}$ & 0.003 & 49.3 & $\begin{array}{c}2.063 \\
(1.350-3.154)^{*}\end{array}$ & 0.296 & 3.3 & & & \\
\hline $\mathrm{N}$ & $213 / 424$ & $\begin{array}{c}0.883 \\
(0.582-1.339)\end{array}$ & - & - & $\begin{array}{c}0.940 \\
(0.530-1.667)\end{array}$ & - & - & & & \\
\hline
\end{tabular}


Table 2C. Results of meta-analysis for rs696 polymorphism in NFKBA and cancer susceptibility.

\begin{tabular}{|c|c|c|c|c|c|c|c|c|c|c|}
\hline \multirow{2}{*}{$\begin{array}{l}\text { Variables } \\
\text { (rs696) }\end{array}$} & \multirow{2}{*}{$\begin{array}{l}\text { Case/ } \\
\text { control }\end{array}$} & \multicolumn{3}{|c|}{ C vs. T } & \multicolumn{3}{|c|}{ CC vs. TT } & \multicolumn{3}{|c|}{ CT vs. TT } \\
\hline & & OR $(95 \% \mathrm{CI})$ & $p^{a}$ & $I^{2}(\%)$ & OR $(95 \% \mathrm{Cl})$ & $p^{a}$ & $I^{2}(\%)$ & OR $(95 \% \mathrm{Cl})$ & $p^{a}$ & $I^{2}(\%)$ \\
\hline Total & $3556 / 5705$ & $\begin{array}{c}0.952 \\
(0.894-1.014)\end{array}$ & 0.121 & 13.8 & $\begin{array}{c}0.891 \\
(0.783-1.013)\end{array}$ & 0.194 & 79.5 & $\begin{array}{c}0.884 \\
(0.738-1.058)\end{array}$ & 0.044 & 24.6 \\
\hline \multicolumn{11}{|l|}{ Ethnicity } \\
\hline Caucasian & $1670 / 1857$ & $\begin{array}{c}0.971 \\
(0.882-1.069)\end{array}$ & 0.034 & 0.0 & $\begin{array}{c}0.930 \\
(0.766-1.128)\end{array}$ & 0.055 & 36.5 & $\begin{array}{c}0.809 \\
(0.676-0.969)^{*}\end{array}$ & 0.459 & 0.0 \\
\hline Asian & $1886 / 3848$ & $\begin{array}{c}0.939 \\
(0.863-1.021)\end{array}$ & 0.431 & 42.6 & $\begin{array}{c}0.861 \\
(0.725-1.023)\end{array}$ & 0.527 & 0.0 & $\begin{array}{c}0.921 \\
(0.691-1.227)\end{array}$ & 0.015 & 45.8 \\
\hline \multicolumn{11}{|c|}{ Source of control } \\
\hline PB & $2018 / 2151$ & $\begin{array}{c}0.963 \\
(0.882-1.051)\end{array}$ & 0.047 & 34.2 & $\begin{array}{c}0.906 \\
(0.759-1.081)\end{array}$ & 0.080 & 27.1 & $\begin{array}{c}0.908 \\
(0.687-1.200)\end{array}$ & 0.050 & 33.4 \\
\hline $\mathrm{HB}$ & $1538 / 3554$ & $\begin{array}{c}0.941 \\
(0.858-1.031)\end{array}$ & 0.397 & 0.0 & $\begin{array}{c}0.875 \\
(0.726-1.054)\end{array}$ & 0.439 & 0.0 & $\begin{array}{c}0.868 \\
(0.656-1.148)\end{array}$ & 0.093 & 28.3 \\
\hline
\end{tabular}

Cancer type

\begin{tabular}{|c|c|c|c|c|c|c|c|c|c|c|}
\hline $\mathrm{HL}$ & $514 / 414$ & $\begin{array}{c}0.792 \\
(0.656-0.956)^{*}\end{array}$ & 0.116 & 34.8 & $\begin{array}{c}0.658 \\
(0.448-0.965)^{*}\end{array}$ & 0.076 & 46.6 & $\begin{array}{c}1.126 \\
(0.408-3.111)\end{array}$ & 0.118 & 34.8 \\
\hline \multirow[t]{3}{*}{ CRC } & $1355 / 2020$ & $\begin{array}{c}1.003 \\
(0.907-1.110)\end{array}$ & 0.269 & 5.7 & $\begin{array}{c}1.014 \\
(0.825-1.2480\end{array}$ & 0.702 & 0.0 & $\begin{array}{c}0.901 \\
(0.673-1.206)\end{array}$ & 0.184 & 16.7 \\
\hline & \multirow{2}{*}{$\begin{array}{l}\text { Case/ } \\
\text { control }\end{array}$} & \multicolumn{3}{|c|}{$\mathrm{CC}+\mathrm{CT}$ vs. TT } & \multicolumn{3}{|c|}{ CC vs. CT+TT } & & & \\
\hline & & OR $(95 \% \mathrm{Cl})$ & $\boldsymbol{p}^{\mathrm{a}}$ & $I^{2}(\%)$ & OR $(95 \% \mathrm{Cl})$ & $\boldsymbol{P}^{\mathrm{a}}$ & $I^{2}(\%)$ & & & \\
\hline Total & $3556 / 5705$ & $\begin{array}{c}0.879 \\
(0.787-0.982)^{*}\end{array}$ & 0.107 & 15.3 & $\begin{array}{c}0.946 \\
(0.811-1.103)\end{array}$ & 0.025 & 29.5 & & & \\
\hline \multicolumn{11}{|l|}{ Ethnicity } \\
\hline Caucasian & $1670 / 1857$ & $\begin{array}{c}0.851 \\
(0.719-1.007)\end{array}$ & 0.304 & 3.1 & $\begin{array}{c}1.002 \\
(0.718-1.399)\end{array}$ & 0.010 & 53.7 & & & \\
\hline Asian & $1886 / 3848$ & $\begin{array}{c}0.901 \\
(0.778-1.044)\end{array}$ & 0.054 & 32.5 & $\begin{array}{c}0.923 \\
(0.798-1.068)\end{array}$ & 0.306 & 2.9 & & & \\
\hline
\end{tabular}

Source of control

\begin{tabular}{cccccccc} 
PB & $2018 / 2151$ & $\begin{array}{c}0.887 \\
(0.765-1.028)\end{array}$ & 0.075 & 28.1 & $\begin{array}{c}0.945 \\
(0.718-1.243)\end{array}$ & 0.016 & 45.0 \\
\hline HB & $1538 / 3554$ & $\begin{array}{c}0.870 \\
(0.736-1.028)\end{array}$ & 0.205 & 11.9 & $\begin{array}{c}0.942 \\
(0.781-1.136)\end{array}$ & 0.165 & 16.9
\end{tabular}

Cancer type

\begin{tabular}{|c|c|c|c|c|c|c|c|}
\hline $\mathrm{HL}$ & $514 / 414$ & $\begin{array}{c}0.758 \\
(0.534-1.077)\end{array}$ & 0.080 & 45.3 & $\begin{array}{c}0.734 \\
(0.562-0.958)^{*}\end{array}$ & 0.347 & 0.0 \\
\hline CRC & $1355 / 2020$ & $\begin{array}{c}0.908 \\
(0.760-1.086)\end{array}$ & 0.726 & 0.0 & $\begin{array}{c}0.995 \\
(0.680-1.456)\end{array}$ & 0.009 & 62.4 \\
\hline
\end{tabular}

$I^{2}-0-25$, means no heterogeneity; 25-50, means modest heterogeneity; >50, means high heterogeneity; HWE - Hardy-Weinberg equilibrium; $\mathrm{Y}$ - polymorphisms conformed to HWE in the control group; $\mathrm{N}$ - polymorphisms did not conform to HWE in the control group; $P^{\mathrm{a}}-P$ value of $\mathrm{Q}$ test for heterogeneity test; ${ }^{*}$ means statistically significant $(P<0.05)$; The source of control, $\mathrm{HB}-$ hospitalbased; PB - population-based; HCC - hepatocellular carcinoma; HL - Hodgkin lymphoma; CRC - colorectal cancer; BC - breast cancer. 
Table 3. Methodological quality of the included studies according to the Newcastle-Ottawa Scale.

\begin{tabular}{|c|c|c|c|c|c|c|c|c|c|c|}
\hline & Author & Ethnicity & $\begin{array}{l}\text { Adequacy } \\
\text { of Case } \\
\text { Definition }\end{array}$ & $\begin{array}{l}\text { Representa- } \\
\text { tiveness of } \\
\text { the Cases }\end{array}$ & $\begin{array}{l}\text { Selection of } \\
\text { Controls }\end{array}$ & $\begin{array}{c}\text { Definition of } \\
\text { Controls }\end{array}$ & $\begin{array}{c}\text { Comparability } \\
\text { Cases/ } \\
\text { Controls }\end{array}$ & $\begin{array}{l}\text { Ascertainment } \\
\text { of exposure }\end{array}$ & $\begin{array}{c}\text { Same } \\
\text { method of } \\
\text { ascertainment }\end{array}$ & $\begin{array}{l}\text { Non- } \\
\text { response } \\
\text { rate }\end{array}$ \\
\hline \multirow[t]{11}{*}{ rs2233406 } & Lu et al. & Asian & * & * & NA & * & $* *$ & * & * & * \\
\hline & Zhang et al. & Asian & * & * & NA & * & ** & * & * & * \\
\hline & Cheng et al. & Asian & * & * & NA & * & ** & * & * & * \\
\hline & Lin et al. & Asian & * & * & NA & * & $* *$ & * & * & * \\
\hline & Tan et al. & Asian & * & * & NA & * & $* *$ & * & * & * \\
\hline & Wang et al. & Asian & * & * & NA & * & $* *$ & * & * & * \\
\hline & Wang et al. & Asian & * & * & NA & * & $* *$ & * & * & * \\
\hline & Wang et al. & Asian & * & * & NA & * & $* *$ & * & * & * \\
\hline & He et al. & Asian & * & * & NA & * & $* *$ & * & * & * \\
\hline & Han et al. & Asian & * & * & NA & * & $* *$ & * & * & * \\
\hline & Umar et al. & Asian & * & * & * & * & $* *$ & * & * & * \\
\hline \multirow[t]{8}{*}{ rs3138053 } & Lin et al. & Asian & * & * & NA & * & $* *$ & * & * & * \\
\hline & Tan et al. & Asian & * & * & NA & * & $* *$ & * & * & * \\
\hline & Gao et al. & Asian & * & * & * & * & $* *$ & * & * & * \\
\hline & Spink et al. & Caucasian & * & * & * & NA & $* *$ & * & * & * \\
\hline & He et al. & Asian & * & * & NA & * & $* *$ & * & * & * \\
\hline & Han et al. & Asian & * & * & NA & * & $* *$ & * & * & * \\
\hline & Zhang et al. & Asian & * & * & * & * & $* *$ & * & * & * \\
\hline & Cheng et al. & Asian & * & * & NA & * & $* *$ & * & * & * \\
\hline \multirow[t]{9}{*}{ rs696 } & Umar et al. & Asian & * & * & * & * & $* *$ & * & * & * \\
\hline & Gao et al. & Asian & * & * & NA & * & $* *$ & * & * & * \\
\hline & Gao et al. & Caucasian & * & * & NA & * & $* *$ & * & * & * \\
\hline & Zhang et al. & Asian & * & * & NA & * & $* *$ & * & * & * \\
\hline & Gao et al. & Asian & * & * & * & * & ** & * & * & * \\
\hline & $\begin{array}{l}\text { Osborne } \\
\text { et al. }\end{array}$ & Caucasian & * & * & * & NA & $* *$ & * & * & * \\
\hline & Chang et al. & Caucasian & * & * & * & NA & $* *$ & * & * & * \\
\hline & Han et al. & Asian & * & * & NA & * & $* *$ & * & * & * \\
\hline & Song et al. & Caucasian & * & * & * & * & $* *$ & * & * & * \\
\hline
\end{tabular}

This table identifies 'high' quality choices with a 'star'. A study can be awarded a maximum of one star for each numbered item within the Selection and Exposure categories. A maximum of two stars can be given for Comparability. ${ }^{*}$, Yes; NA - not applicable. (http://www.ohri.ca/programs/clinical_epidemiology/oxford.htm).

\section{3}

Indexed in: [Current Contents/Clinical Medicine] [SCI Expanded] [ISI Alerting System] [ISI Journals Master List] [Index Medicus/MEDIINE] [EMBASE/Excerpta Medica] [Chemical Abstracts/CAS] [Index Copernicus] 


\begin{tabular}{|c|c|c|c|}
\hline Study ID & & OR $(95 \% \mathrm{Cl})$ & $\%$ weight \\
\hline Spink et al. (2006) & $\rightarrow$ & $2.05(1.51,2.79)$ & 12.50 \\
\hline He et al. (2009) & & $\rightarrow \quad 41.05(29.58,56.97)$ & 12.48 \\
\hline Lin et al. (2012) & $\rightarrow$ & $7.87(5.75,10.52)$ & 12.51 \\
\hline Tan et al. (2013) & $\rightarrow$ & $4.72(3.35,6.65)$ & 12.46 \\
\hline Cheng et al. (2013) & & $\rightarrow \quad 29.17(20.02,42.50)$ & 12.42 \\
\hline Zhang et al. (2014) & & $\rightarrow 59.49(45.84,77.21)$ & 12.55 \\
\hline Gao et al. (2014) & & $12.04(8.43,17.18)$ & 12.45 \\
\hline Han et al. (2015) & + & $2.81(2.41,3.28)$ & 12.63 \\
\hline Overall $(\mathrm{I}$-squared $=98.9 \%, \mathrm{p}=0.000)$ & & $10.754 .18,27.70)$ & 100.00 \\
\hline Note: Weights are from random effects analysis & & & \\
\hline .013 & & 77.2 & \\
\hline
\end{tabular}

\begin{tabular}{|c|c|c|}
\hline Study ID & OR $(95 \% \mathrm{Cl})$ & $\%$ weight \\
\hline Osborne et al. (2005) & $\rightarrow 2.240 .(63,7.97)$ & 0.50 \\
\hline Gao et al. (2006) & $1.09(0.68,1.75)$ & 5.01 \\
\hline Gao et al. (2006) & $0.88(0.55,1.42)$ & 5.36 \\
\hline Chang et al. (2006) & $0.69(0.47,0.99)$ & 10.42 \\
\hline Song et al. (2011) & $0.88(0.71,1.09)$ & 27.75 \\
\hline Umar et al. (2013) & $0.72(0.49,1.07)$ & 8.97 \\
\hline Zhang et al. (2014) & $0.64(0.46,0.89)$ & 12.13 \\
\hline Gao et al. (2014) & $1.25(0.88,1.87)$ & 8.40 \\
\hline Han et al. (2015) & $0.94(0.75,1.19)$ & 21.46 \\
\hline Overall (I-squared $=39.1 \%, \mathrm{p}=0.107)$ & $0.88(0.79,0.98)$ & 100.00 \\
\hline .125 & 7.97 & \\
\hline
\end{tabular}

\begin{tabular}{|c|c|c|}
\hline Study ID & OR $(95 \% \mathrm{Cl})$ & $\%$ weight \\
\hline \multicolumn{3}{|l|}{$\mathrm{N}$} \\
\hline Wang et al. (2014) & $0.08(0.00,1.24)$ & 1.49 \\
\hline Wang et al. (2014) & $1.11(0.69,1.80)$ & 16.09 \\
\hline Wang et al. (2014) & $1.53(0.65,3.59)$ & 9.83 \\
\hline Subtotal (I-squared $=57.7 \%, p=0.094)$ & $1.01(0.42,2.40)$ & 27.41 \\
\hline \multicolumn{3}{|l|}{ Y } \\
\hline He et al. (2009) & $0.22(0.03,1.61)$ & 2.71 \\
\hline Lin et al. (2012) & $2.85(0.89,9.16)$ & 6.54 \\
\hline Cheng et al. (2013) & $1.94(0.35,10.70)$ & 3.60 \\
\hline Tan et al. (2013) & $1.62(0.52,5.03)$ & 6.83 \\
\hline Umar et al. (2013) & $1.19(0.64,2.20)$ & 13.55 \\
\hline Zhang et al. (2014) & $1.41(0.58,3.44)$ & 9.31 \\
\hline Han et al. (2015) & $2.61(1.68,4.05)$ & 16.92 \\
\hline Lu et al. (2015) & $1.05(0.56,1.99)$ & 13.14 \\
\hline Subtotal (I-squared $=42.0 \%, p=0.099)$ & $1.54(1.03,2.30)$ & 72.59 \\
\hline Overall $(I-$ squared $=46.7 \%, p=0.043)$ & $1.39(0.98,1.98)$ & 100.00 \\
\hline \multicolumn{3}{|l|}{ Note: Weights are from random effects analysis } \\
\hline .0046 & 217 & \\
\hline
\end{tabular}

Figure 2A. Meta-analysis of the association between NFKBIA rs3138053 polymorphism and overall cancer susceptibility (C vs. T).

Figure 2B. Meta-analysis of the association between NFKBIA rs696 polymorphism and overall cancer susceptibility $(\mathrm{CC}+\mathrm{CT}$ vs. TT $)$.
Figure $2 \mathrm{C}$. Subgroup analysis of the association between NFKBIA polymorphisms and overall cancer risk by HWE (HardyWeinberg equilibrium). 


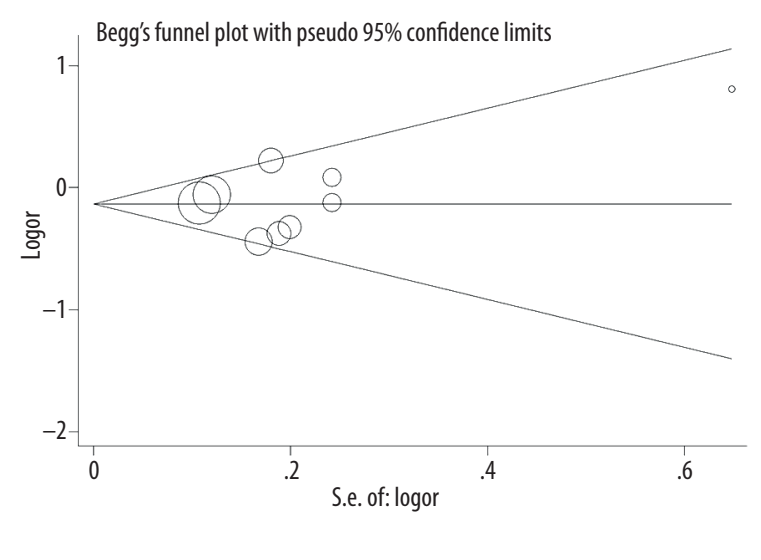

Figure 3A. Publication bias examinations by Begg's funnel plots and Egger's test (rs696: CC+CT vs. TT). Asymmetrical plots or $\mathrm{P}<0.10$ suggest potential bias. Each point represents a separate study for the indicated association, and the horizontal line depicts mean effect size. Logor natural logarithm of OR; s.e. standard error.

susceptibility and severity in sporadic colorectal cancer and oral cancer. Klein et al. [42] proved that the polymorphism of NFKBIA was linked to Crohn's disease. Other studies also drew similar conclusions in breast [9], prostate [13], and stomach [14] cancers. However, results from studies in various geographic areas were not consistent. To the best of our knowledge, this is the first meta-analysis to assess the relationship between these 3 polymorphisms (rs2233406, rs3138053, and rs696) of NFKBIA gene and overall cancer susceptibility. Our analysis validated that individuals with the variant allele (rs3138053) appear to have increased susceptibility to cancer. Interestingly, we found a contrary effect of variant allele (rs696), which seems to be associated with decreased susceptibility to cancer. In the subgroup analysis by cancer type, significantly decreased susceptibility of Hodgkin lymphoma with rs696 polymorphism was observed, whereas no significant association was found among studies of colorectal cancer.

The heterogeneity test in the present study showed that there was no evident heterogeneity in terms of the 3 polymorphisms for all cancer types between the studies. Additionally, various cancer categories did not contribute to the overall heterogeneity in association with the polymorphisms, suggesting that our present combined analyses were unbiased, regardless of

\section{References:}

1. Domingo-Domenech J, Oliva C, Rovira A et al: Interleukin 6, a nuclear factorkappaB target, predicts resistance to docetaxel in hormone-independent prostate cancer and nuclear factor-kappaB inhibition by PS-1145 enhances docetaxel antitumor activity. Clin Cancer Res, 2006; 12(18): 5578-86

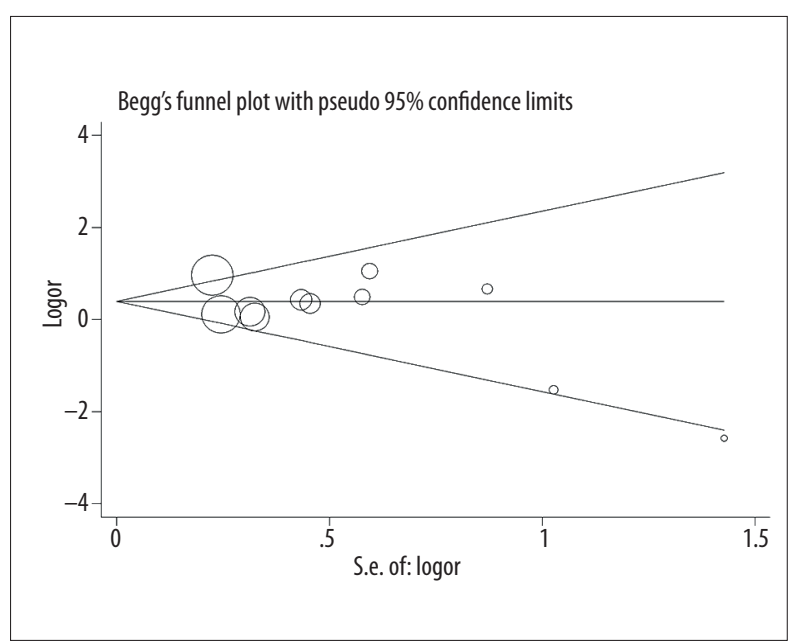

Figure 3B. Publication bias examinations by Begg's funnel plots and Egger's test (rs2233406: CC vs. CT+TT). Asymmetrical plots or $\mathrm{P}<0.10$ suggest potential bias. Each point represents a separate study for the indicated association, and the horizontal line depicts mean effect size. Logor natural logarithm of OR; s.e. standard error.

cancer types. Despite the obvious advantages of our metaanalysis containing large sample sizes, some limitations of this study should be mentioned. The complex factors such as age, sex, and region may bring some bias. Studies reported in other languages may bias the present results because the negative findings are usually difficult to be included. Therefore, further study is needed to evaluate the independent and combined effect of these polymorphisms.

\section{Conclusions}

In conclusion, this meta-analysis indicated that the rs 3138053 polymorphism of NFKBIA gene is a candidate for susceptibility to overall cancers, especially in HCC cancer, while the rs696 plays a protective role against cancers, especially in Hodgkin lymphoma patients and in Caucasians. Moreover, because of the limitations described above, well-designed studies considering gene-gene and gene-environment effects should be conducted to confirm these relationships.

\section{Conflict of interest statement}

None. 
4. Lind DS HS, Malaty J, Rekkas S et al: Nuclear factor-kappa B is upregulated in colorectal cancer. Surgery, 2001; 130: 363-69

5. Oltulu YM, Coskunpinar E, Ozkan G et al: Investigation of NF-kappaB1 and NF-kappaBIA gene polymorphism in non-small cell lung cancer. Biomed Res Int, 2014; 2014: 530381

6. Yang JJ, Cho LY, Ko KP et al: Interaction effects between genes involved in the AKT signaling pathway and phytoestrogens in gastric carcinogenesis: a nested case-control study from the Korean Multi-Center Cancer Cohort. Mol Nutr Food Res, 2012; 56(11): 1617-26

7. Shiels MS, Engels EA, Shi J et al: Genetic variation in innate immunity and inflammation pathways associated with lung cancer risk. Cancer, 2012 118(22): 5630-36

8. Huang D, Yang L, Liu Y et al: Functional polymorphisms in NFkappaB1/ IkappaBalpha predict risks of chronic obstructive pulmonary disease and lung cancer in Chinese. Hum Genet, 2013; 132(4): 451-60

9. Curran JE, Weinstein SR, Griffiths LR: Polymorphic variants of NFKB1 and its inhibitory protein NFKBIA, and their involvement in sporadic breast cancer. Cancer Lett, 2002; 188(1-2): 103-7

10. Lewander A, Arbman G, Sun XF: Polymorphism in the promoter region of the NFKBIA gene is rare in Swedish and Chinese colorectal cancer patients and controls. Mol Med Rep, 2010; 3(1): 69-74

11. White KL, Vierkant RA, Phelan CM et al: Polymorphisms in NF-kappaB inhibitors and risk of epithelial ovarian cancer. BMC Cancer, 2009, 9: 170

12. Shafi'i MSM SS, Mustapha MA: The genetic variation A>G at 3' UTR of nuclear factor kappa B 1 A (NFkB1A) influences susceptibility of sporadic colorectal cancer in Malaysian population. Int Med J, 2012; 19(2): 98-101

13. Zhang $P$, Wei $Q$, Li $X$ et al: A functional insertion/deletion polymorphism in the promoter region of the NFKB1 gene increases susceptibility for prostate cancer. Cancer Genet Cytogenet, 2009; 191(2): 73-77

14. Lo SS, Chen JH, Wu CW, Lui WY: Functional polymorphism of NFKB1 promoter may correlate to the susceptibility of gastric cancer in aged patients. Surgery, 2009; 145(3): 280-85

15. Gao J, Pfeifer D, He L-J et al: Association ofNFKBIApolymorphism with colorectal cancer risk and prognosis in Swedish and Chinese populations. Scand J Gastroenterol, 2007; 42(3): 345-50

16. Bodelon C, Madeleine MM, Johnson LG et al: Genetic variation in the TLR and NF-kappaB pathways and cervical and vulvar cancer risk: a population-based case-control study. Int J Cancer, 2014; 134(2): 437-44

17. Bu H, Rosdahl I, Sun XF, Zhang H: Importance of polymorphisms in NFkappaB1 and NF-kappaBlalpha genes for melanoma risk, clinicopathological features and tumor progression in Swedish melanoma patients. J Cance Res Clin Oncol, 2007; 133(11): 859-66

18. Marcos M, Pastor I, Gonzalez-Sarmiento R, Laso FJ: A functional polymorphism of the NFKB1 gene increases the risk for alcoholic liver cirrhosis in patients with alcohol dependence. Alcohol Clin Exp Res, 2009; 33(11): 1857-62

19. Kim LH, Shin HD, Park BL et al: Identification of variants in NFKBIA and association analysis with hepatocellular carcinoma risk among chronic HBV patients. Hum Mutat, 2003; 21(6): 652-53

20. Higgins JP, Thompson SG, Deeks JJ, Altman DG: Measuring inconsistency in meta-analyses. BMJ, 2003; 327(7414): 557-60

21. Mantel N, Haenszel W: Statistical aspects of the analysis of data from retrospective studies of disease. J Natl Cancer Inst, 1959; 22(4): 719-48

22. DerSimonian R, Laird N: Meta-analysis in clinical trials. Controll Clin Trials, 1986; 7(3): 177-88

23. Chang ET, Birmann BM, Kasperzyk JL et al: Polymorphic variation in NFKB1 and other aspirin-related genes and risk of Hodgkin lymphoma. Cancer Epidemiol Biomarkers Prev, 2009; 18(3): 976-86
24. Cheng CW, Su JL, Lin CW et al: Effects of NFKB1 and NFKBIA gene polymorphisms on hepatocellular carcinoma susceptibility and clinicopathological features. PloS One, 2013; 8(2): e56130

25. Gao J, Xu HL, Gao S et al: Genetic polymorphism of NFKB1 and NFKBIA genes and liver cancer risk: a nested case-control study in Shanghai, China. BMJ Open, 2014; 4(2): e004427

26. Han X, Zhang JJ, Yao N et al: Polymorphisms in NFKB1 and NFKBIA Genes Modulate the Risk of Developing Prostate Cancer among Han Chinese. Med Sci Monit, 2015; 21: 1707-15

27. He Y, Zhang H, Yin J et al: IkappaBalpha gene promoter polymorphisms are associated with hepatocarcinogenesis in patients infected with hepatitis B virus genotype C. Carcinogenesis, 2009; 30(11): 1916-22

28. Lin CW, Hsieh YS, Hsin CH et al: Effects of NFKB1 and NFKBIA gene polymorphisms on susceptibility to environmental factors and the clinicopathologic development of oral cancer. PloS One, 2012; 7(4): e35078

29. Lu ZH, Gu XJ, Shi KZ et al: Association between genetic polymorphisms of inflammatory response genes and the risk of ovarian cancer. J Formos Med Assoc, 2015 [Epub ahead of print]

30. Osborne J, Lake A, Alexander FE et al: Germline mutations and polymorphisms in the NFKBIA gene in Hodgkin lymphoma. Int J Cancer, 2005; 116(4) 646-51

31. Song S, Chen D, Lu J et al: NFkappaB1 and NFkappaBIA polymorphisms are associated with increased risk for sporadic colorectal cancer in a southern Chinese population. PloS One, 2011; 6(6): e21726

32. Spink CF, Gray LC, Davies FE et al: Haplotypic structure across the I kappa $B$ alpha gene (NFKBIA) and association with multiple myeloma. Cancer Lett, 2007; 246(1-2): 92-99

33. Tan SC, Suzairi MS, Aizat AA, Aminudin MM et al: Gender-specific association of NFKBIA promoter polymorphisms with the risk of sporadic colorectal cancer. Med Oncol, 2013; 30(4): 693

34. Umar M, Upadhyay R, Kumar S et al: Association of common polymorphisms in TNFA, NFkB1 and NFKBIA with risk and prognosis of esophageal squamous cell carcinoma. PloS One, 2013; 8(12): e81999

35. Wang Z, Liu Q-L, Sun W et al: Genetic polymorphisms in inflammatory response genes and their associations with breast cancer risk. Croat Med J, 2014; 55(6): 638-46

36. Zhang Q: Effect of functional nuclear factor kappaB genetic polymorphisms on hepatitis $B$ virus persistence and their interactions with viral mutations on the risk of hepatocellular carcinoma. Annals of Oncology Advance Access, 2014

37. Sun Z, Andersson R: NF-kappaB activation and inhibition: a review. Shock, 2002; 18(2): 99-106

38. Charbonneau B, Block MS, Bamlet WR et al: Risk of ovarian cancer and the NF-kappaB pathway: genetic association with IL1A and TNFSF10. Cancer Res, 2014; 74(3): 852-61

39. Wang $S$, Zhang $M$, Zeng Z et al: IkappaBalpha polymorphisms were associated with increased risk of gastric cancer in a southern Chinese population: a case-control study. Life Sci, 2011; 88(17-18): 792-97

40. Li RN, Hung YH, Lin CH et al: Inhibitor IkappaBalpha promoter functional polymorphisms in patients with rheumatoid arthritis. J Clin Immunol, 2010; 30(5): 676-80

41. Zou YF YF, Feng XL, Tao JH, Ding N: Association between NFKB1-94ins/deIATTG promoter polymorphism and cancer risk: a metaanalysis. Cancer Invest, 2011; 29: 78-85

42. Klein W, Tromm A, Folwaczny C et al: A polymorphism of the NFKBIA gene is associated with Crohn's disease patients lacking a predisposing allele of the CARD15 gene. Int J Colorectal Dis, 2004; 19(2): 153-56 